\title{
A NOTE ON SEMI-GROUPS OF UNBOUNDED SELF-ADJOINT OPERATORS
}

\section{A. DEVINATZ ${ }^{1}$}

In this note we extend a theorem about integral representations of semi-groups of bounded self-adjoint operators to a theorem about semi-groups of unbounded self-adjoint operators. The theorem for semi-groups of bounded self-adjoint operators has been proved in different ways by B. v. Sz. Nagy [4; 5], E. Hille [3], and also follows from a more general theorem of S. Bochner [1]. Semi-groups of unbounded self-adjoint operators arose in quite a natural way in an investigation of the author [2] on positive definite functions.

In the following theorem $D_{A}$ shall represent the domain of an operator $A$ which is defined in a Hilbert space.

THEOREM. Let $\left\{T_{x}\right\}$, for $x>0$, be a semi-group of self-adjoint operators acting in a Hilbert space, i.e. $T_{x_{1}} T_{x_{2}}=T_{x_{1}+x_{2}}$. Further, suppose that for all $f \in \bigcap_{x>0} D_{T_{x}},\left(T_{x} f, f\right)$ is either a bounded or measurable function of $x$ in some interval. Then there exists a unique resolution of the identity $\left\{E_{t}\right\}$ such that $E_{t}=0$ for $t<0$ and

$$
T_{x}=\int_{0}^{\infty} t^{x} d E_{t}
$$

Proof. It has been shown by Sz. Nagy $[5$, p. 73] that the conditions on $\left(T_{x} f, f\right)$ given in the theorem imply that this is a continuous function of $x$ for all $x>0$.

Let us also use one more idea due to Sz. Nagy [5, p. 73]. Let $\left\{E_{t}\right\}$ be the canonical resolution of the identity of $T_{1}$. By the semi-group property we know that $T_{1} \geqq 0$. Since every positive self-adjoint operator has a unique positive self-adjoint square root, we have

$$
T_{1 / 2}=\int_{0}^{\infty} t^{1 / 2} d E t
$$

Proceeding in this way and using the semi-group property we must get for all positive integers $m$ and $n$ that

$$
T_{m / 2^{n}}=\int_{0}^{\infty} t^{m / 2^{n}} d E_{t}
$$

Received by the editors June 15, 1953.

1 National Science Foundation Fellow. 
Let us now consider the operators

$$
H_{x}=\int_{0}^{\infty} t^{x} d E_{t}
$$

By the discussion above $H_{m / 2^{n}}=T_{m / 2^{n}}$. Now, there exists a countable set of mutually orthogonal manifolds $\left\{M_{k}\right\}_{1}^{\infty}$, whose direct sum is the whole space and such that for all $x>0, H_{x}=\prod_{k=1}^{\infty} \times H_{x}^{(k)}$, where $H_{x}^{(k)}$ is a bounded self-adjoint operator on $M_{k}$ and is the restriction of $H_{x}$ to $M_{k}$ (Sz. Nagy [5, p. 49]). That is to say, $f \in D_{H_{x}}$ if and only if $\sum_{k=1}^{\infty}\left\|H_{x}^{(k)} f_{k}\right\|^{2}<\infty$, where $f_{k} \in M_{k}$ and $f=\sum_{k=1}^{\infty} f_{k}$. Then $H_{x}$ $=\sum_{k=1}^{\infty} H_{x}^{(k)} f_{k}$.

Given any $x>0$ there exists a rational number $m / 2^{n} \geqq x$. From the semi-group property we know then that $D_{T_{m / 2^{n}}} \subseteq D_{T_{x}}$. Therefore for every $f_{k} \in M_{k}, f_{k} \in D_{T_{m / 2^{n}}}$ and therefore belongs to $D_{T_{x}}$. Consequently, since $T_{m / 2^{n}}=H_{m / 2^{n}}$ and by the continuity of $\left(T_{x} f_{k}, f_{k}\right)$ and $\left(H_{x} f_{k}, f_{k}\right)$ as functions of $x$, we must have $T_{x} f_{k}=H_{x}^{(k)} f_{k}=H_{x} f_{k}$. This implies that $T_{x}=H_{x}$ (Sz. Nagy $[5$, p. 35]).

The theorem for two parameter semi-groups offers no difficulty.

\section{REFERENCES}

1. S. Bochner, Completely monotone functions in partially ordereds paces, Duke Math. J. vol. 9 (1942) pp. 519-526.

2. A. Devinatz, Integral representations of positive definite functions, Trans. Amer. Math. Soc. vol. 74 (1953) pp. 56-77.

3. E. Hille, Notes on linear transformations. II. Analyticity of semi-groups, Ann. of Math. (2) vol. 40 (1939) pp. 1-47.

4. B. v. Sz. Nagy, Bedingungen fïr die Multiplikationstabelle eines in sich abgeschlossenen orthogonalen Funktionensystems, Annali di Pisa vol. 6 (1937) pp. 211-224.

5. - Spektraldarstellung linear Transformationen des Hilbertschen Raumes, Ergebnisse der Mathematik und ihrer Grenzgebiete, no. 5, 1942.

The Institute for Advanced Study 